\title{
Isolating the Detrapping of Deuterium in Heavy Ion Damaged Tungsten via Partial Thermal Desorption
}

\author{
M. J. Simmonds ${ }^{\mathrm{a}}$, T. Schwarz-Selinger ${ }^{\mathrm{b}}$, J. H. Yu ${ }^{\mathrm{a}}$, M. J. Baldwin ${ }^{\mathrm{a}}$, R. P. \\ Doerner $^{\mathrm{a}}$, G. R. Tynan ${ }^{\mathrm{a}, \mathrm{c}}$ \\ ${ }^{a}$ Center for Energy Research, UC San Diego, 9500 Gilman Dr., La Jolla, CA \\ 92093-0417, USA \\ ${ }^{b}$ Max-Planck-Institut für Plasmaphysik, Boltzmannstrasse 2, D-85748 Garching, \\ Germany \\ ${ }^{c}$ Department of Mechanical and Aerospace Engineering (MAE), UC San Diego, 9500 \\ Gilman Dr., La Jolla, CA 92093-0411, USA
}

\begin{abstract}
Partial Thermal Desorption Spectrometry (pTDS) progressively depopulated trapped deuterium (D) from heavy-ion damaged tungsten (W) to determine spatial location and detrapping energies. W samples were prepared identically: $5 \mathrm{MeV} \mathrm{Cu}^{2+}$ damaging ions (0.12 peak dpa dose) before $\mathrm{D}_{2}$ plasma loading $\left(10^{24} \mathrm{D} / \mathrm{m}^{2}\right.$ fluence) held at $373 \mathrm{~K}$. Each sample reached one of six pTDS peak-and-hold temperatures. Nuclear Reaction Analysis (NRA) measured the D spatial profile remaining after pTDS, before final TDS.

NRA and TDS measured total D retention were in good agreement. NRA displayed three zones of D-populated defects: (I) near-surface (below $0.1 \mu \mathrm{m}$ ), (II) heavy-ion damage (peaked $\sim 1 \mu \mathrm{m}$ ), and (III) uniform intrinsic (bulk). $\mathrm{D}$ concentration in zone I reduced by $\sim 97 \%$ in samples with pTDS at $597 \mathrm{~K}$ and higher, indicating near-surface traps have low detrapping energy. The Stopping and Range of Ions in Matter (SRIM) predicts a displacement profile
\end{abstract}

Email address: msimmonds@eng.ucsd.edu (M. J. Simmonds) 
for zone II that coincided with measured D profile for samples with pTDS at $597 \mathrm{~K}$ and higher. Samples prepared with pTDS below $597 \mathrm{~K}$ display a distinctly different $\mathrm{D}$ profile in zone II. The complete cycle of $\mathrm{D}_{2}$ plasma loading, pTDS, NRA, and final TDS was modeled with Tritium Migration Analysis Program (TMAP) using a recently developed Pseudo Trap and Temperature Partition (PTTP) scheme. Differences in TDS profiles isolated traps that release between consecutive pTDS temperatures, demonstrating 6 distinct release peaks. The best fit was found with detrapping energies near 1.0, $1.2,1.4,1.6,1.8$, and $1.9 \mathrm{eV}$. These results show that heating at $762 \mathrm{~K}$ for 2.5 hours released $\sim 99 \%$ of retained $\mathrm{D}$ in heavy-ion damaged $\mathrm{W}$. Keywords: Tungsten, Deuterium, Retention, Heavy ion damage, Partial Thermal Desorption, Peak-and-hold, NRA, TDS 
1

2

\section{Introduction}

The retention of tritium in Plasma Facing Materials (PFMs) is an important issue due to both safety concerns as well as maintaining the fuel cycle in fusion devices [1]. The production of fusion neutrons will lead to the degradation of PFMs throughout the bulk of the material. In order to study the effects of neutron damage and tritium exposure, heavy ion damage and deuterium are used as proxies, respectively. The guidelines for the use of heavy ions to simulate neutron damage are outlined in [2].

Experiments conducted to study displacement damaged W typically use NRA and TDS to quantify D retention. NRA probes the D distribution across the near-surface region up to depths of $\sim 10 \mu \mathrm{m}$, whereas TDS measures the released D from throughout the bulk. NRA can not differentiate between trapped and solute $\mathrm{D}$ atoms located between $\mathrm{W}$ lattice sites, nor can it directly determine detrapping energy or trap type. Among other factors such as surface recombination, the release peaks for thermally desorbed D from $\mathrm{W}$ are correlated to both the spatial position and detrapping energy of a particular type of defect. The measured release rate from the surface is the result of the escape of $\mathrm{D}$ from multiple types of defects, each having a particular detrapping energy, followed by subsequent diffusion through the material to reach the surface where recombination occurs. The resulting TDS data may display one or more effective release peaks, due to nearby detrapping energies from multiple defects as well as multiple D fill levels for a single defect type.

Comparing the activation energies calculated with Density Functional Theory (DFT) and attained by modeling thermal desorption data, various 
defect types in $\mathrm{W}$ have been associated with a particular dominant detrapping energy [3]. Inferred detrapping energies range for dislocations and grain boundaries from 0.8 to $1.0 \mathrm{eV}$, vacancies from 1.3 to $1.7 \mathrm{eV}$, and vacancy clusters from 1.8 to $2.4 \mathrm{eV}$. Detrapping energies may also overlap. Scanning transmission electron microscopy [4] suggests dislocation loops span 0.4 to $1.8 \mathrm{eV}$ [5]. Though DFT predicts multiple D filling of some defects (e.g. mono-vacancies $[6,7])$ the individual release peaks may not be directly resolvable in TDS data if the detrapping energies are too close together. Note that the values determined from TDS data are dependent on both the attempt frequency, generally assumed to be $10^{13} \mathrm{~s}^{-1}[7]$, as well as the value used to model surface recombination.

Previous studies $[3,8,9]$ have used varied sample temperatures during plasma exposure to selectively populate defects. At higher sample temperature, defects with lower detrapping energies can not be effectively filled with $\mathrm{D}$ if the release rate is large relative to the trapping rate. The increased temperature may significantly influence the evolution of defects to be populated, complicating the interpretation of such experiments. For instance, mono-vacancies may be partially annealed as they recombine with mobile interstitials, and between $523-573 \mathrm{~K}$ the vacancies become mobile and can further annihilate or agglomerate into clusters [10,11]. Positron annihilation spectroscopy shows that the clusters grow in samples heated to $650 \mathrm{~K}$ in under 5 minutes [12]. Thus, at high sample temperatures, the assumption of a static population of defects during plasma exposure may no longer be valid. In addition, the increased diffusivity of D at higher temperature may increase retention as deeper intrinsic traps become filled. This results in the 
broadening of each release peak. Therefore the direct comparison of samples prepared with various sample temperatures during plasma exposure is not straightforward.

To better quantify the spatial location and detrapping energies associated with various defects, we devised an experimental approach to sequentially depopulate each defect according to detrapping energy. Whereas previous experimental studies that attempted to model NRA and TDS data have assumed specific detrapping energies and spatial concentrations, this approach aims to constrain both quantities and test if a discrete detrapping energy model can reproduce the experimental data. Similar to the approach by Efimov et al. [13], all damaged and D decorated samples have the same initial conditions prior to pTDS. Unlike Efimov, by holding the sample at a fixed elevated temperature (peak-and-hold) after the pTDS linear ramp, defects with appreciable release rates at that temperature will further depopulate. The released D then either travels further into the material, filling traps with higher detrapping energy, or reaches the surface to escape the sample. NRA is carried out after each pTDS procedure to provide a spatial profile of the remaining $\mathrm{D}$ atom concentration. The resulting data allows us to then infer the spatial profile with respect to detrapping energies and densities from each spatial zone attributed to plasma-induced, heavy ion beam induced, and intrinsic trap sites.

Typically the TDS temperature ramp is assumed fast enough to neglect defect evolution, while the pTDS peak-and-hold may change the defect population. Since pTDS temperatures in this experiment span 467-762 K, mono-vacancies may become mobile but vacancy clusters would not dissoci- 
ate $(\sim 1200 \mathrm{~K}[14])$. Additionally, traps may agglomerate migrating defects that can result in an increase to the detrapping energy.

In a similar experiment, Zhao et al. [15] performed pTDS on W samples with 0.01 and 1 displacements per atom (dpa). The resulting full TDS profiles show that the initial release for the $0.01 \mathrm{dpa}$ sample starts at a temperature well above the peak-and-hold and slightly above initial release for the $1 \mathrm{dpa}$ sample. Here it is worth noting that the full TDS of the $0.01 \mathrm{dpa}$ sample does not exhibit obvious defect evolution due to the pTDS. On the other hand, the 1 dpa does show that the highest temperature release peak significantly grew, likely due to vacancy agglomeration during the peak-and-hold.

In some previous experiments, within the heavy ion damage region there is a discrepancy between some experimental NRA data and SRIM predicted damage profiles. NRA data from plasma exposure at elevated sample temperatures ( $\sim 600 \mathrm{~K}$ and above) $[8,9,12,16]$ do not display a significant near-surface peak and are spatially commensurate with the SRIM calculated dpa profile. Yet lower sample temperature during plasma exposure yields a $\mathrm{D}$ concentration in the near-surface that can not be explained by plasma induced defects alone. The deviation in trap profile and consequently D concentration measurements is likely due to a different spatial profile for low detrapping energy defects.

\section{Experiment}

\subsection{Sample Preparation}

W samples originated from a certified 99.95 wt.\% powder metallurgy polycrystalline rod, $6 \mathrm{~mm}$ in diameter and cut into disks $1.5 \mathrm{~mm}$ thick. 
The plasma facing surface received a mirror-like finish by successive polish treatments ending with a $3 \mu \mathrm{m}$ grit. Arithmetic mean surface roughness, measured with a confocal microscope, resulted in $28 \pm 5 \mathrm{~nm}$ after polish. Contaminants from polishing were removed in successive ultrasonic baths of acetone followed by ethanol. Next, the samples were annealed at $1173 \mathrm{~K}$ for 1 hour in a vacuum chamber below $10^{-4} \mathrm{~Pa}$. As noted in [17], a broken W sample displayed elongated grains perpendicular to the surface with dimension on the order of $10 \mu \mathrm{m}$ parallel to the surface, as viewed by a scanning electron microscope. The maximum annealing temperature is well below the recrystallization temperature, leaving an intrinsic level of various defects throughout the bulk of the sample.

\subsection{Heavy Ion Damage}

The previously prepared $\mathrm{W}$ samples were irradiated with $5.0 \mathrm{MeV} \mathrm{Cu}^{2+}$ ions at the TOF beamline of the tandem accelerator laboratory at MaxPlanck-Institut für Plasmaphysik in Garching (IPP) while under a low vacuum of $10^{-6} \mathrm{~Pa}$ and held at $295 \mathrm{~K}$ with a water-cooled sample holder. Details of the setup can be found in [18]. The beam had a full width at half maximum of $2 \mathrm{~mm}$, and was rastered over the samples to achieve lateral homogeneity. The implanted $\mathrm{Cu}$ dose was $1.215 \times 10^{18}$ ions $/ \mathrm{m}^{2}$ with an average flux of $2.4 \times 10^{15}$ ions $/ \mathrm{m}^{2} / \mathrm{s}$, which maintained an impurity level below the intrinsic Cu level [19]. In accordance with Stoller et al. [20], the "Quick" KinchinPease option and a displacement damage threshold of $90 \mathrm{eV}$ were used to calculate the displacement profile in SRIM shown in Fig. 1 with a peak dpa of 0.12. According to the simulation, on average each energetic $\mathrm{Cu}$ ion is estimated to produce a collision cascade with over 5,600 W lattice displace- 
ments. This sample temperature during plasma exposure was low enough to avoid the annealing of displacement damage that we have reported in previous work [17].

\section{3. $D_{2}$ Plasma Exposure}

One undamaged sample, as well as the identically prepared $\mathrm{Cu}$ ion irradiated samples, were exposed to $\mathrm{D}_{2}$ plasma with a neutral pressure of $0.7 \mathrm{~Pa}$ in the PISCES-E device, a plasma etcher with a $13.56 \mathrm{MHz}$ RF source [21]. The air-cooled sample holder was negatively biased to implant D with an ion impact energy of $110 \mathrm{eV}$ and maintained the sample at $373 \mathrm{~K}$ as measured by a thermocouple in contact with the rear of the sample. An RF compensated Langmuir probe inferred an average flux $\left(\Gamma_{\text {incident }}\right)$ of $1.8 \times 10^{20}$ ions $/ \mathrm{m}^{2} / \mathrm{s}$ uniformly across the surface of the sample holder as detailed in [22]. Each sample received a total fluence of $10^{24} \mathrm{D} / \mathrm{m}^{2}$ after $\sim 1.5$ hours.

\subsection{Partial TDS}

The plasma-exposed samples were kept at room temperature for 25 days before being subjected to pTDS. Samples were mounted on the tip of a thermocouple within a vacuum below $10^{-6} \mathrm{~Pa}$. Parabolic mirrors focused the heat from infrared lamps on the sample surface. A programmable controller was set to heat the samples at a constant rate of $0.5 \mathrm{~K} / \mathrm{s}$ before plateauing for 2.5 hours at a particular peak-and-hold temperature. That is, instead of completely desorbing the sample as in a typical TDS run by heating the samples to a peak temperature near $1300 \mathrm{~K}$, temperatures well below that were chosen to selectively depopulate the corresponding lower energy traps. In what follows, the label for each pTDS sample refers to this peak-and-hold 
temperature (e.g. "pTDS at $467 \mathrm{~K}$ "). It should be noted that two control samples were not thermally desorbed at this stage, one without pTDS labeled "No pTDS" and one without heavy ion damage, "No $\mathrm{Cu}$ ".

\subsection{NRA}

NRA was performed on each sample at IPP Garching 20 days after the pTDS procedure. The $\mathrm{D}\left({ }^{3} \mathrm{He}, \mathrm{p}\right){ }^{4} \mathrm{He}$ nuclear reaction measured the depth profiles of the remaining D concentration as prescribed by Mayer with a detector positioned at a scattering angle of $135^{\circ}$ [23]. A ${ }^{3} \mathrm{He}$ ion beam was used to probe the first $\sim 6 \mu \mathrm{m}$ of $\mathrm{D}$ implanted in $\mathrm{W}$ with decreasing energies of $3.5,2.5,2.0,1.65,1.5,1.3,1.1,0.8$, and $0.6 \mathrm{MeV}$. Both the energy spectra of the resultant protons and alphas were captured in solid state detectors to determine the depth distribution of the retained D. Relative to the protons, the resulting alpha particles are only detectable for low ${ }^{3} \mathrm{He}$ beam energies since the energy of reflected ${ }^{3} \mathrm{He}$ ions overlap with the alpha energy. Probing with a ${ }^{3} \mathrm{He}$ energy slightly below and above the cross section peak (i.e. 0.6 and $0.8 \mathrm{MeV}$ ) enhances the near-surface resolution of $\mathrm{D}$ concentration. In addition to detecting both product ions, the various sources of straggle are minimized with relatively low probing energies. As a result, we can resolve D trapped within the near-surface $(\sim 100 \mathrm{~nm})$ region; deeper NRA results have poorer spatial resolution. Using the NRA data, both SimNRA and NRADC were then employed to determine the most probable $\mathrm{D}$ concentration as a function of depth $[23,24]$. 


\subsection{Final TDS}

A further 18 days elapsed between NRA and the final TDS run where all samples were heated with a constant $0.5 \mathrm{~K} / \mathrm{s}$ ramp rate up to a peak temperature above $1300 \mathrm{~K}$ to ensure full desorption of $\mathrm{D}$. The partial pressures of $\mathrm{H}_{2}, \mathrm{HD}$, and $\mathrm{D}_{2}$ were measured with a quadrupole mass spectrometer (QMS). The thermally desorbed particle flux was calculated by converting the QMS measured partial pressure via a calibrated $\mathrm{D}_{2}$ leak. The total $\mathrm{D}$ flux was calculated as described in further detail by $\mathrm{Yu}$ [25], as the sum of the HD and twice the $\mathrm{D}_{2}$ flux. Note that the HD flux was calibrated to the $\mathrm{D}_{2}$ leak, without any further correction for ionization efficiency. Since the detection efficiency is expected to increase for ions of lighter mass this procedure leads to a small overestimation of the total D flux. However, as HD contributed only an average of $28 \%$ to the total D flux this is of minor importance. As described previously [17], variable ambient laboratory humidity, temperature, and length of vacuum break influence the water content adsorbed to the TDS chamber walls. This leads to a significant $\mathrm{H}_{2}$ background signal that was scaled to and subtracted from the raw $\mathrm{HD}$ and $\mathrm{D}_{2}$ signals.

\section{Results}

After the pTDS procedure was applied to each sample, and prior to final TDS, the spatial profile of D concentration in each sample was measured with NRA. In Fig. 1, the experimental data displays a monotonic decrease in D concentration as the pTDS peak-and-hold temperature is increased. The estimated error determined by NRADC as a concentration range is indicated as the corresponding transparent colored region in the figure. The NRA pro- 
files have distinct spatial zones, labeled I-III respectively: zone I composed of the near-surface $(\sim 0.1 \mu \mathrm{m})$ region, zone II composed of the $\mathrm{Cu}$ damage $(\sim 1 \mu \mathrm{m})$ region, and zone III composed of the bulk of the samples depth. Within zone I, the control sample without heavy ion beam damage (solid black) displays a peak D concentration near 1.5 at. \% that decays exponentially with a characteristic length of $0.05 \mu \mathrm{m}$. The intrinsic defects left after sample preparation annealing (below the recrystallization temperature) are assumed to have a uniform distribution throughout the bulk, zone III. For $\mathrm{Cu}$ ion beam damaged samples, the dominant contribution to total $\mathrm{D}$ retention is seen in zone II. The D concentrations for pTDS temperatures of $525 \mathrm{~K}$ and higher largely coincide with the SRIM predicted damage profile (dot-dashed orange). The "No pTDS" and the $467 \mathrm{~K}$ pTDS samples have profiles that are more heavily weighted closer to the surface. The small, but measurable, D level near $2 \mu \mathrm{m}$ suggests the D diffusion front reached beyond the SRIM profile, which was confirmed by subsequent TMAP modeling that predicted the front reached $\sim 20 \mu \mathrm{m}$.

In Fig. 2, the surface flux of $\mathrm{D}$ atoms released from the $\mathrm{W}$ samples during each pTDS and final TDS are shown as dashed and solid lines respectively. The $700 \mathrm{~K}$ pTDS sample is not shown due to an experimental error with the initial temperature ramp. The pTDS profiles (dashed lines) display the sequential removal of D from traps with increasing pTDS temperature, and exhibit a sharp drop in released D flux when fixed at their peak-and-hold temperature. This D flux plotted against time instead of temperature displays an exponential decay during the pTDS peak-and-hold (not shown here but similar to [15]), indicating the depopulation of traps that exhibit a signif- 


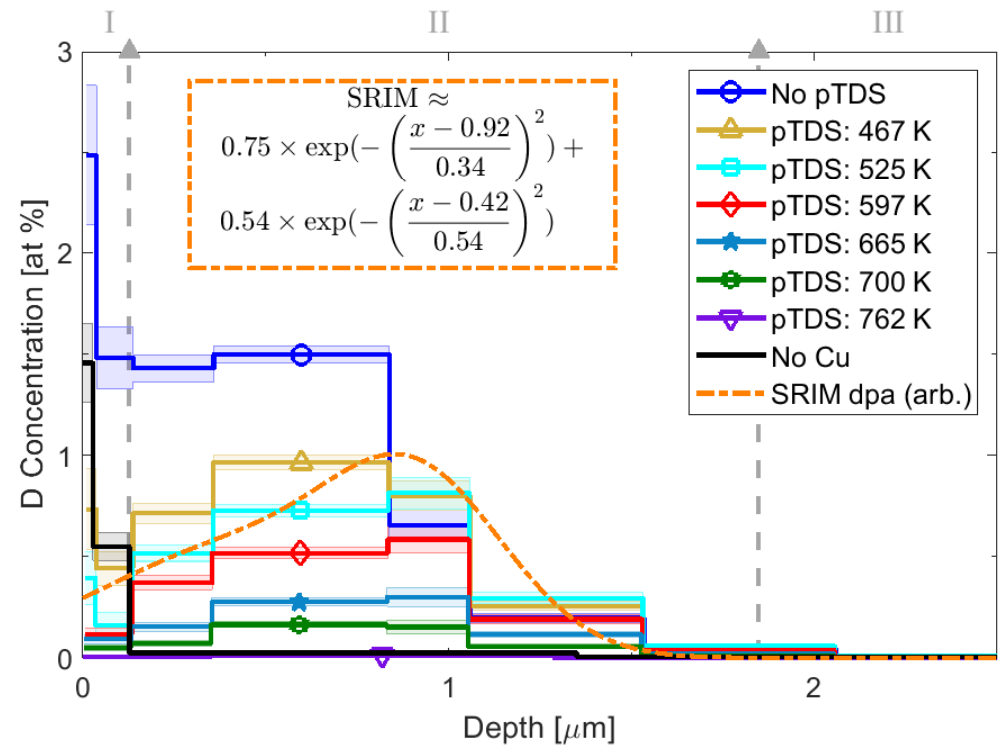

Figure 1: The D concentration measured through NRA decreases with increasing pTDS temperature. The SRIM predicted trap profile (dot-dashed orange) for $5 \mathrm{MeV} \mathrm{Cu}{ }^{2+}$ displays a peak near $\sim 0.9 \mu \mathrm{m}$ and shown here scaled to 1 . Note that the experimental dose resulted in a calculated peak dpa of 0.12. A two Gaussian fit to the SRIM profile is defined by the normalized function. Dashed silver vertical lines indicate the approximate transition between zones I-III.

icant release probability at the pTDS peak-and-hold temperature. The first and weakest D filled traps are highly sensitive to surface conditions and storage time in between D implantation and TDS $[26,27]$. Whereas the pTDS samples were thermally desorbed after $\sim 1$ month, the control samples waited $\sim 2$ months. That extra storage time likely led to the "No pTDS" control (dark blue) having a lower first peak than in the other $\mathrm{Cu}$ damaged samples. The "No pTDS" control sample shows a significant increase in the amount of released D as compared to the "No Cu" control sample (black) for all temperatures below $1000 \mathrm{~K}$. The initial release of the "No pTDS" control sample 

as illustrated in the following discussion.

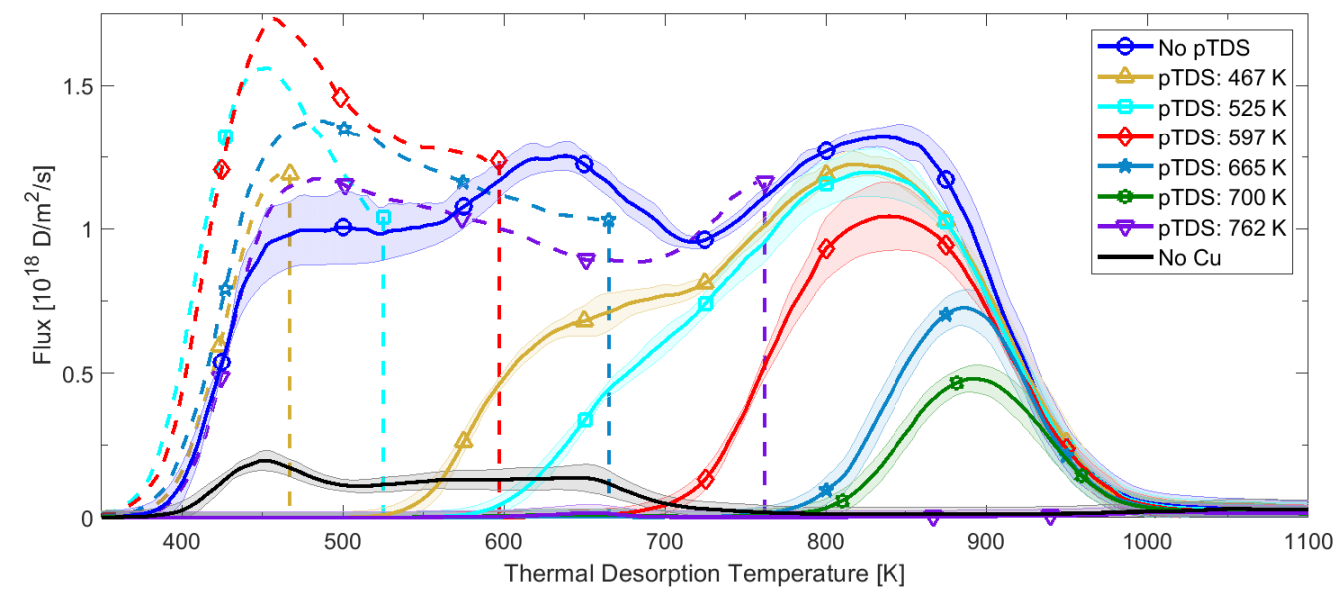

Figure 2: The D surface flux during pTDS (dashed) and the final TDS (solid) are plotted with respect to a $0.5 \mathrm{~K} / \mathrm{s}$ linear heating ramp. Once pTDS samples reached a specific peakand-hold temperature, the flux during the $2.5 \mathrm{hr}$ peak-and-hold decays exponentially in time and approaches zero at this fixed temperature.

239 it appears that the pTDS peak-and-hold temperature is at least $40 \mathrm{~K}$ lower than the leading edge of the initial release temperature during the subsequent final TDS. For example, the sample with a pTDS peak-and-hold temperature of $467 \mathrm{~K}$ (dashed gold) only begins to appreciably release D when the temperature exceeds $510 \mathrm{~K}$ during the subsequent final TDS (solid gold). This separation indicates that the underlying assumptions of the PTTP scheme [28] can be used to distinguish and isolate traps with progressively higher release energies. As a result, taken together the NRA and TDS data can be used to quantify the location and concentration of D residing in each trap,

Note that in Fig. 1, the NRA profiles for the control samples labeled

begins near the $373 \mathrm{~K}$ plasma exposure sample temperature. For all cases, 
"No Cu" and "No pTDS" have a high D concentration located in zone I. In the "No $\mathrm{Cu}$ " case this zone I population forms the majority of retained D inventory, while for the "No pTDS" case (which did suffer $\mathrm{Cu}$ ion beam damage) the zone I retained population sits on top of a significant retained D inventory located deeper in zone II. Comparing the "No pTDS" and the $467 \mathrm{~K}$ and $525 \mathrm{~K}$ pTDS cases in Fig. 1, we observe that the zone I retained D inventory is largely reduced, and is undetectable for the $597 \mathrm{~K}$ pTDS case shown in red in Fig. 1. Furthermore, the "No Cu" (solid black) and $597 \mathrm{~K}$ (solid red) final TDS curves in Fig. 2 display a crossing near $700 \mathrm{~K}$. These observations show that the majority of the "No $\mathrm{Cu}$ " sample D inventory that was trapped in zone I has been completely released at $700 \mathrm{~K}$ whereas the ion beam damaged sample that underwent a $597 \mathrm{~K}$ pTDS treatment is just beginning to release $\mathrm{D}$ at this temperature. Considering Fig. 1 and Fig. 2 results together, we can then conclude that the zone I traps are induced by the plasma and have relatively low detrapping energies that release at temperatures below 700 K. Furthermore, we can conclude that most of the increased inventory in ion beam damaged samples is located in zone II and releases at higher temperatures. These two experimental observations suggest that the NRA, pTDS, and final TDS approach used here may permit the inference of both spatial distribution of traps with differing trapping energies. We take up a deeper examination of the utility of the combined NRA, pTDS, and final TDS data sets after examining the self-consistency of the overall retention data emerging from these combined techniques.

By integrating the NRA profile over the depth into the material we can determine the remaining retained D inventory for each pTDS case. The 
result of this analysis is shown by the black circle symbols in Fig. 3, and shows a gradual reduction in the retained D as the pTDS peak-and-hold temperature is increased. Likewise, by integrating the pTDS release histories shown by the dashed curves in Fig. 2, we can determine how much D was released for each pTDS case. The result of this analysis is shown by the filled blue upright triangle data points in Fig. 3, and shows a gradual increase in the amount of released D with increasing pTDS peak-and-hold temperature. Finally, integrating the final TDS release curves shown by the solid curves in Fig. 2, we can determine the remaining D inventory throughout the bulk of the material. The result, shown as the inverted red triangles in Fig. 3 shows a gradual decrease in retained D inventory as the pTDS peak-andhold temperature is increased, and is in good quantitative agreement with the NRA results. The sum of the pTDS and TDS data (purple diamonds) provides an independent measurement of retained inventory and shows a nearly constant value of $(12 \pm 1) \times 10^{20} \mathrm{D} / \mathrm{m}^{2}$, consistent with the data point obtained from the damaged control sample ("No pTDS" in Fig. 1 and 2) which is plotted at $373 \mathrm{~K}$ on the $\mathrm{x}$-axis in Fig. 3. Thus the independent NRA, pTDS, and final TDS techniques give consistent retained inventory measurements.

\section{Isolating Detrapping Energies}

The simulation of the pTDS, NRA, and final TDS stages are well constrained by both the identical initial conditions and the controlled depopulation of each trap. Here we assume each trap concentration and spatial profile as well as the filling thereof during D implantation are the same for 


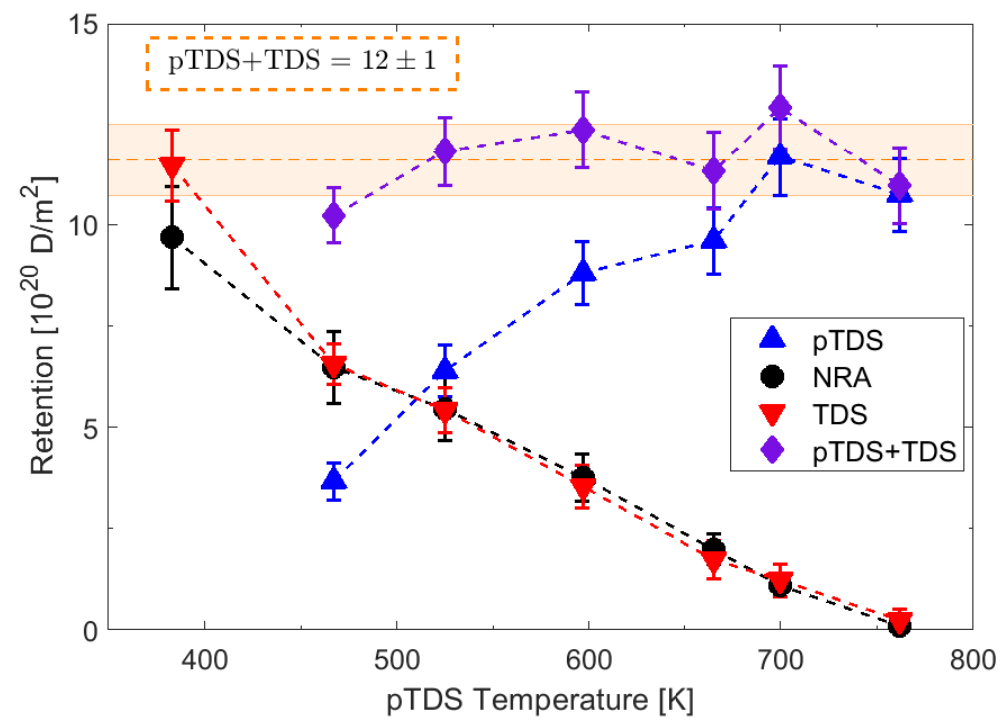

Figure 3: The sum total D retention measured in the first $5.5 \mu \mathrm{m}$ through NRA and the bulk through TDS. Plotted at the plasma exposure sample temperature of $373 \mathrm{~K}$, the "No pTDS" sample shows the largest deviation from NRA. The sum of D retention measured from pTDS and TDS is consistent as seen by the average (orange shaded region). Dashed lines are only shown to guide the eye.

all samples prior to the application of the pTDS procedure. In what follows, we exploit the selective depopulation of traps with lower detrapping energies in order to isolate the spatial location and detrapping energy of the desorbed D. In order to do this, for a given NRA profile or pTDS release dataset, we subtract the next highest pTDS temperature dataset (i.e. consecutive pTDS temperatures). The difference then can be used to determine spatial location (from differential NRA data) or release temperatures (from differential final TDS data). Furthermore, the spatial location of a given trap energy will be described by a spatial profile function $\left(f_{k}^{j}(x)\right)$ that corresponds to the spatial distribution of the D that was released during consecutive pTDS exposures; 
we will find that this function will be zone $(j)$ and trap type $(k)$ dependent.

\subsection{NRA Subtraction}

The NRA D profiles shown in Fig. 1 display a distinctly different shape for pTDS samples subjected to peak-and-hold temperatures of $525 \mathrm{~K}$ and above. Fig. 2 shows the remaining $\mathrm{D}$ in these higher temperature pTDS samples are trapped in defects with increasing detrapping energy. Fig. 4 displays the concentration difference profiles $(\Delta C)$ computed as the difference between consecutive NRA D profiles and labeled by the two pTDS temperatures used to produce a given profile. The difference profiles for these higher temperature pTDS samples and the $762 \mathrm{~K}$ pTDS sample display a peak at a depth of 0.7-1.0 $\mu \mathrm{m}$, in agreement with the SRIM predicted dpa profile (dot-dashed orange), showing that the computed dpa distribution at a given depth is correlated to the difference concentration at that location. We therefore use the SRIM predicted spatial profile to define the spatial profile function $f_{\text {high }}^{I I}(x)$ for zone II, where the trap type $(k=h i g h)$ refers to high detrapping energies associated with higher temperature release.

In Fig. 5, the D concentration profile for the "No $\mathrm{Cu}$ " sample (black) displays a large near-surface component in zone I due to $\mathrm{D}_{2}$ plasma exposure (note the much larger concentration scale compared to Fig. 4). The profile exponentially decays into the bulk as seen in previous work $[29,30]$. A fit (dot-dashed red) to the "No $\mathrm{Cu}$ " is shown as an intrinsic concentration plus an exponential decay with characteristic length of $0.05 \mu \mathrm{m}$. The exponential fit to the plasma induced trap distribution in zone I is then chosen to define the spatial distribution $f_{\text {low }}^{I}(x)$, where "low" refers to low detrapping energy traps (i.e. Fig. 4 shows $f_{\text {high }}^{I}(x)$ equals zero). 


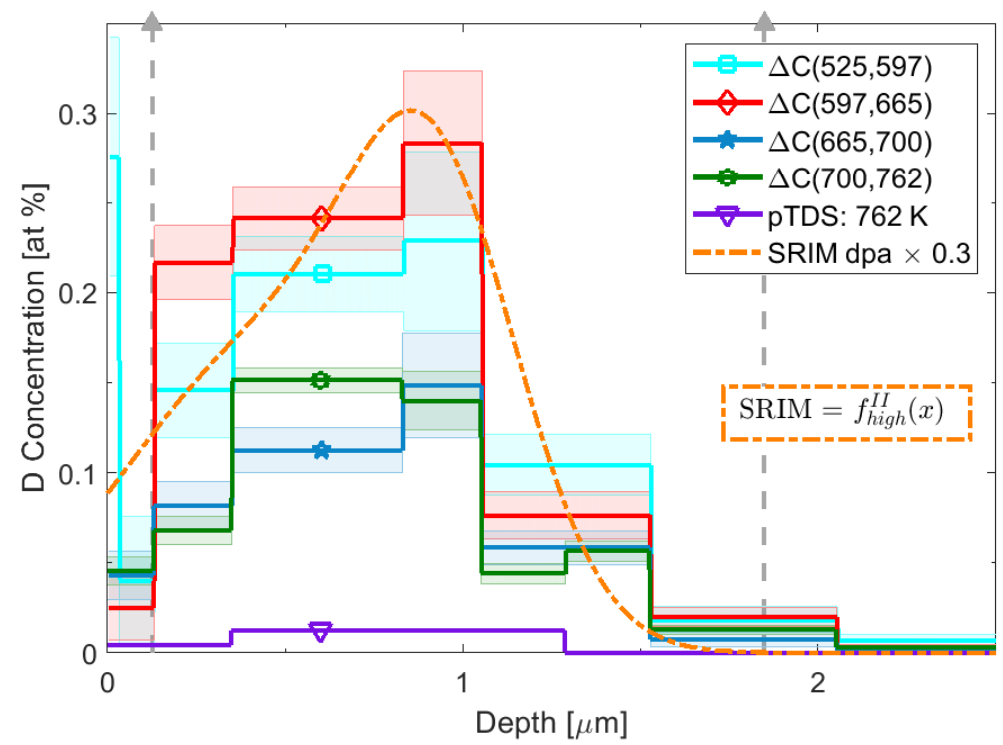

Figure 4: The difference in D concentration between consecutive pTDS samples are shown as solid lines. The damage profile of zone II is further defined and fit well by the SRIM dpa profile scaled to 0.3 for ease of comparison (dot-dashed orange).

In order to isolate and determine the spatial location of low energy traps in zone II, both the "No Cu" and $597 \mathrm{~K}$ pTDS profiles are subtracted from the "No pTDS" profile (solid blue). First, noting the similarity in D profile for zone I between the "No Cu" and "No pTDS" NRA data shown in Fig. 1, we assume the plasma induced traps are represented by the "No $\mathrm{Cu}$ " profile. Second, the samples with pTDS peak-and-hold temperature at $597 \mathrm{~K}$ and above display no additional zone I component. Lastly, as shown in Fig. 4, these higher temperature pTDS samples display a D concentration coincident with heavy ion induced traps located in zone II. Thus the remaining D found after subtracting both the "No $\mathrm{Cu}$ " and $525 \mathrm{~K}$ pTDS reveals the profile for heavy ion induced traps associated with lower detrapping energies. 


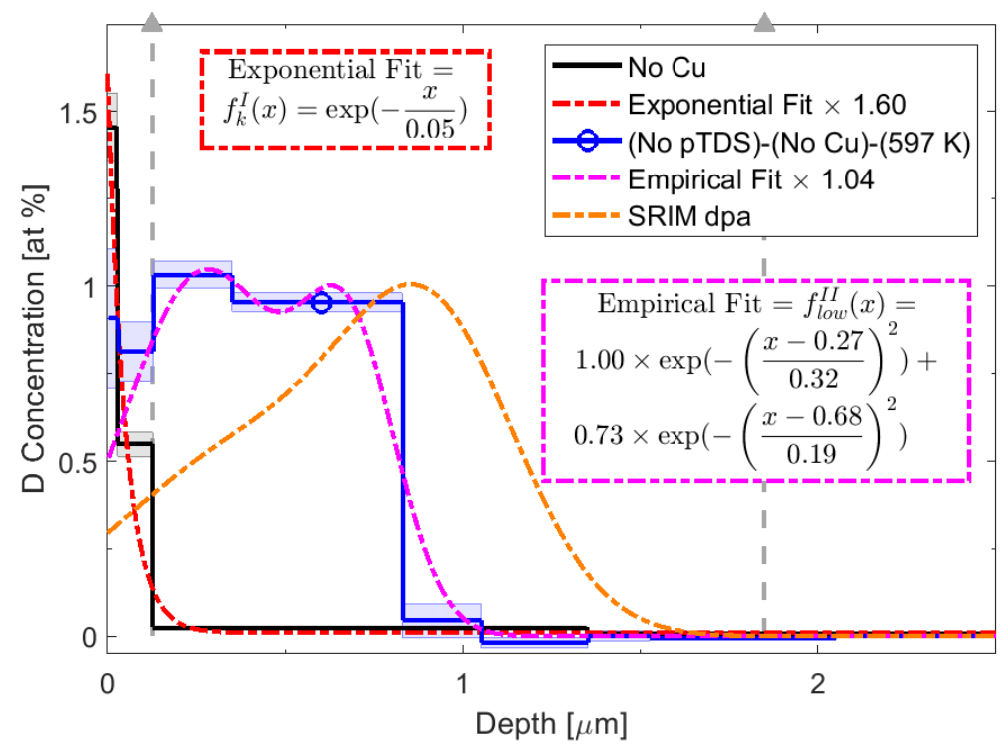

Figure 5: The "No Cu" sample is shown as solid black with an exponential decay function (dot-dashed red). The solid blue line representing "No pTDS," subtracting both the "No $\mathrm{Cu}$ " and $597 \mathrm{~K}$ pTDS NRA profiles, isolates the low energy traps due to heavy ion damage. An empirical fit (dot-dashed magenta) to this D profile is significantly shallower than the SRIM profile (dot-dashed orange).

An empirical fit to this difference $\left(f_{\text {low }}^{I I}(x)\right)$ is shown in Fig.5 as the sum of two Gaussians (dot-dashed magenta), though the two distinct features may simply be an artifact of the NRA resolution. This difference-concentration distribution is quite distinct from both the near-surface zone I concentration profile, and the trapped D located within higher detrapping energy traps found in Fig. 4.

\subsection{TDS Subtraction}

Similarly, the differences between the final TDS data obtained from consecutive pTDS peak-and-hold temperature runs are shown in Fig. 6. Using 
the difference-flux $(\Delta \Gamma)$ TDS profiles, we can infer the minimum number of distinct traps in damaged W. The first difference (dark blue) is too broad to be due to a single trap but would appear to have at least two detrapping energies. The separation and width of each additional difference suggest at least four more individual detrapping energies. Note that the overlapping initial release of the last two differences (turquoise and green) is likely due to the same detrapping energy. Lastly, the highest pTDS temperature at $762 \mathrm{~K}$ (purple) may have a unique detrapping energy of its own as further evidenced by the corresponding NRA profile in Fig. 4. Thus, there are likely at least 6 or 7 unique detrapping energies needed to model this data set obtained with a sample temperature of $373 \mathrm{~K}$ during plasma exposure, as seen in previous work [28, 13, 31].

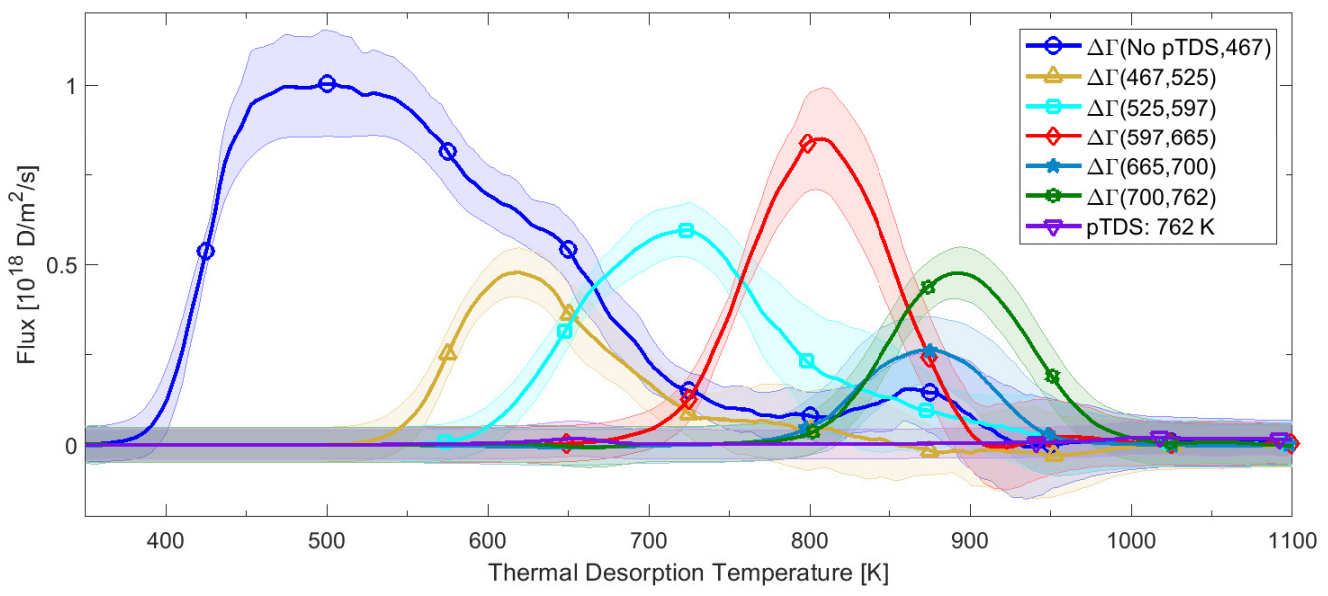

Figure 6: The difference in D flux during final TDS $(\Delta \Gamma)$ for each consecutive pTDS sample pair are shown. The first dark blue line displays a broader primary peak than the other differences. Each of the remaining differences can be modeled with a single detrapping energy. All of the differences, except for the green line, begin to escape the sample at a unique temperature. 
These difference datasets obtained from NRA and final TDS measurements can be used to constrain a reaction-diffusion model of the D in damaged $\mathrm{W}$ to determine the spatial distribution and trapping energy that best reproduces these observations. We take up this modeling effort in the following.

\section{TMAP Simulation with PTTP}

In order to use the verified and validated reaction-diffusion code TMAP7 $[32,33,34]$ to model these results that require more than three types of traps with distinct detrapping energies, we employed a Pseudo Trap and Temperature Partitioning (PTTP) scheme as outlined and applied to TMAP7 [28]. In brief, for a particular temperature range this scheme utilizes a pseudo trap that accounts for multiple traps with deep energy wells that do not appreciably release but may act to trap mobile D from solute. When applied to TMAP7, only three traps are modeled at a time: the two lowest detrapping energy traps are included and can act to both trap and detrap D atoms, and a pseudo trap (which models all traps with higher detrapping energies) is included and primarily acts to trap D atoms.

Reaction-diffusion parameters as outlined in $[28,26]$ were used and detailed in Table 1. During the $\mathrm{D}_{2}$ plasma exposure phase, the implanted $\mathrm{D}$ flux was simulated as a Gaussian with mean depth $\left(r_{\text {implant }}\right)$ and standard deviation $\left(\sigma_{\text {implant }}\right)$ with a reflection coefficient $(R)$.

The heavy ion damage, together with the intrinsic defects leftover after sample preparation and annealing act as the initial trap concentrations for 
Table 1: Summary of fixed parameters and activation energies $\left(E_{a}\right)$ used in the TMAP simulation.

\begin{tabular}{c|lcc|r} 
Ref. & Parameter & & $E_{a}[\mathrm{eV}]$ \\
\hline \hline$[6]$ & $D_{s}$ & $1.6 \times 10^{-7}$ & {$\left[\mathrm{~m}^{2} / \mathrm{s}\right]$} & 0.25 \\
{$[35]$} & $K_{r}$ & $3 \times 10^{-25} T^{-1 / 2}$ & {$\left[\mathrm{~m}^{4} / \mathrm{s}\right]$} & -2.06 \\
\hline$[7]$ & $\nu_{0}$ & $10^{13}$ & {$[1 / \mathrm{s}]$} & \\
{$[36]$} & $R$ & 0.65 & \\
{$[36]$} & $r_{\text {implant }}$ & 4 & {$[\mathrm{~nm}]$} & \\
& \multirow{2}{*}{$\sigma_{\text {implant }}$} & 2 & {$[\mathrm{~nm}]$} & \\
\cline { 2 - 3 } & \multicolumn{2}{|r}{} & &
\end{tabular}

zone II and III respectively. Since TMAP7 uses a static trap concentration, here we also take the plasma induced trap profile in zone I as an initial condition. The formation of plasma induced defects is postulated to be due to a solute D saturated region stressing the near-surface zone I [29]. As detailed in [37], equation 1 estimates the diffusion limited D solute maximum concentration during D implantation. Using the parameters from Table 1 and sample temperature $(\mathrm{T})$ during plasma exposure gives a $\mathrm{D}$ saturation level that approaches $2 \times 10^{-4}$ at. \% . TMAP simulations show that within $1 \%$ of total exposure time $(<1 \mathrm{~min})$, the solute concentration has reached this saturation value. Compared to the total plasma exposure time of over an hour, the time required for plasma to induce these traps through saturation of zone I is relatively short and simply approximated as an initial trap concentration.

$$
C_{s, \text { max }} \approx \frac{(1-R) \cdot \Gamma_{\text {incident }} \cdot r_{\text {implant }}}{n_{W} \cdot D_{s}(T)}
$$


Equation 2 defines the total initial trap concentration $\left(C^{o}(x)\right)$ as a summation over trap type and spatial zone. Each trap type $(k)$ is identified by a distinct detrapping energy $\left(E_{k}\right)$. Note that the traps are ordered according to increasing detrapping energy. Each spatial zone $(j=\mathrm{I}$, II, or III) has an associated profile function $\left(f_{k}^{j}(x)\right)$, that is chosen according to the inferred trap distributions found from experimental data and shown in Fig. 4 and 5. All the profile functions are normalized to a peak value of unity. The profile function is multiplied by the peak concentration $\left(C_{k}^{j}\right)$ for each trap and zone. For $j=\mathrm{I}$, the exponential fit detailed in Fig. 5 defines the profile function. For $j=$ III, the profile function is simply a constant. We also differentiate the particular trap profile in zone II for low and high energy traps as seen in Fig. 4 and 5. For $j=$ II, the lower detrapping energies use the empirical fit shown in Fig. 5 for zone II, while the higher detrapping energies use the SRIM profile. As a result of constraining the profile functions by experiment, the peak concentration $\left(C_{k}^{j}\right)$ for each trap $(k)$ in each zone $(j)$ and the detrapping energies $\left(E_{k}\right)$ become the free parameters that can be adjusted within TMAP7 in order to fit the experimental NRA and TDS data. Note that the sample preparation and D implantation phase are assumed identical for all samples in this dataset, thus we need only model this part of the experiment once for a given set of free parameters.

$$
\begin{aligned}
C^{o}(x) & =\sum_{k} C_{k}^{o}(x) \\
& =\sum_{k}\left[C_{k}^{I} f_{k}^{I}(x)+C_{k}^{I I} f_{k}^{I I}(x)+C_{k}^{I I I} f_{k}^{I I I}(x)\right]
\end{aligned}
$$




\subsection{Annealing Defects}

While the assumption of a static trap concentration is likely valid for a relatively fast temperature ramp (e.g. $0.5 \mathrm{~K} / \mathrm{s}$ ), the peak-and-hold during pTDS may allow for significant trap evolution. Sakurada et al. [38] presents D retention data from post damage ( $6 \mathrm{MeV}$ Fe, $0.1 \mathrm{dpa}$ ) annealed W. Noting the similarity of damaging species, energy, and dpa level as well as the clear TDS release peaks, we inferred the reduced trap fraction $\left(\delta_{k}\right)$ with respect to each TDS release peak. Table 2 summarizes the reduction values at several anneal temperatures $\left(T_{\text {anneal }}\right)$ and a linear interpolation determined the reduction value at each pTDS temperature. In this simplified model, traps are removed (e.g. Frenkel pair recombination), while defect agglomeration is neglected. Once again, since TMAP7 uses a static trap concentration, the removal of defects may be modeled incrementally. The first 30 minutes of the peak-and-hold duration was broken into 100 equal time increments and at the end of each increment a hundredth of the estimated reduced fraction $\left(0.01 \delta_{k}\right)$ was removed from the trap population. D was conserved as filled traps were removed by moving the excess D to solute concentration. Including trap removal provides the best agreement with experimental data and are the parameters presented in Table 2 .

\subsection{Fitting Results}

The complete cycle of $\mathrm{D}_{2}$ plasma exposure, pTDS, and final TDS was simulated for each sample and all free parameters were optimized utilizing the technique of simulated annealing [39]. During the simulated annealing, the free parameters were constrained according to the differences in consecutive TDS and NRA data previously outlined. The simulation of a particular 
Table 2: Summary of the best fit parameters for each trap $(k)$. The peak concentrations $\left(C_{k}^{j}\right)$ in each zone $(j)$ and the detrapping energies $\left(E_{k}\right)$ modeled using the PTTP scheme. Reduced trap fraction $\left(\delta_{k}\left(T_{\text {anneal }}\right)=1-C_{k}\left(T_{\text {anneal }}\right) / C_{k}(300)\right)$ for each annealing temperature in Kelvin estimated from [38].

\begin{tabular}{cccccc|ccc}
$k$ & $C_{k}^{I}$ & $C_{k}^{I I}$ & $f_{k}^{I I}(x)$ & $C_{k}^{I I I}$ & $E_{k}$ & $\delta_{k}(300)$ & $\delta_{k}(573)$ & $\delta_{k}(873)$ \\
\hline \hline 1 & 0.80 & 0.26 & Empirical & 0.0001 & 1.01 & 0 & 1 & 36 \\
2 & 1.18 & 0.28 & Empirical & 0.0022 & 1.19 & 0 & 44 & 63 \\
3 & 0.45 & 0.43 & Empirical & 0.0020 & 1.39 & 0 & 32 & 51 \\
4 & 0.34 & 0.15 & SRIM & 0.0019 & 1.57 & 0 & 44 & 61 \\
5 & 0.00 & 0.48 & SRIM & 0.0008 & 1.76 & 0 & 8 & 50 \\
6 & 0.00 & 0.23 & SRIM & 0.0000 & 1.93 & 0 & 0 & 2 \\
\hline & {$[$ at.\%] } & {$[$ at.\%] } & Profile & {$[$ at.\%] } & {$[\mathrm{eV}]$} & {$[\%]$} & {$[\%]$} & {$[\%]$}
\end{tabular}

set of trap parameters results in unique NRA and TDS profiles. Both the experimental and simulation data were interpolated to a finely spaced grid to directly compare the "goodness of fit." The Normalized Root Mean Square Error (NRMSE) as well as the Normalized Mean Absolute Error (NMAE), defined by the absolute difference between experiment and simulation, were the metrics used to determine the optimal fit. In addition, the resulting NRMSE and NMAE were weighted with respect to their total D retention and added together to determine a single fit metric. For instance, the "No pTDS" sample had the highest $\mathrm{D}$ retention and had the largest weight while the highest temperature pTDS at $762 \mathrm{~K}$ contributed the least weight when determining the best fit parameters. 
The number of traps used to simulate the experiment was varied from 5 to 9. As outlined in the section on TDS subtraction, the best fit was found with 6 traps with detrapping energies near 1.0, 1.2, 1.4, 1.6, 1.8, and $1.9 \mathrm{eV}$. Using 7 traps, nearly the same energies were found and one additional energy of $2.4 \mathrm{eV}$ produced nearly the same fit metric. The highest pTDS sample at $762 \mathrm{~K}$ may either be due to a partially released $1.9 \mathrm{eV}$ trap or a filled $2.4 \mathrm{eV}$ trap. The experimental TDS release temperature appears significantly higher than a $1.9 \mathrm{eV}$ trap. By $762 \mathrm{~K}$, an appreciable amount of vacancy agglomeration may have occurred during the peak-and-hold that is not modeled but may account for the higher temperature release. We also note that the results shown are based on the PTTP scheme that assumes a minimal separation in detrapping energies. For instance, a difference below $0.1 \mathrm{eV}$ for consecutive detrapping energies leads to a significant error as outlined in [28]. Table 2 provides a summary of the resulting trap concentrations and energies found to give the best fit to the NRA and pTDS data with the least number of traps. The total trap concentration, $C^{o}(x)$, is shown in Fig. 7 (solid black) together with the $k^{\text {th }}$ trap concentration profiles, $C_{k}^{o}(x)$, given as the dotdashed lines. The measured NRA profile from the "No pTDS" sample is also shown and has a shape that overlays with the $C^{o}(x)$ total trap concentration profile.

The simulated NRA profiles and final TDS release histories (dashed lines) are compared to the experimental data (solid lines) in Fig. 8 and 9 respectively. The simulated NRA and TDS produced reasonable fits with normalized fit metric values of $\sim 0.7$ for both. A possible reason for the discrepancy may be due to using detrapping energies separated by at least $0.1 \mathrm{eV}$. For 


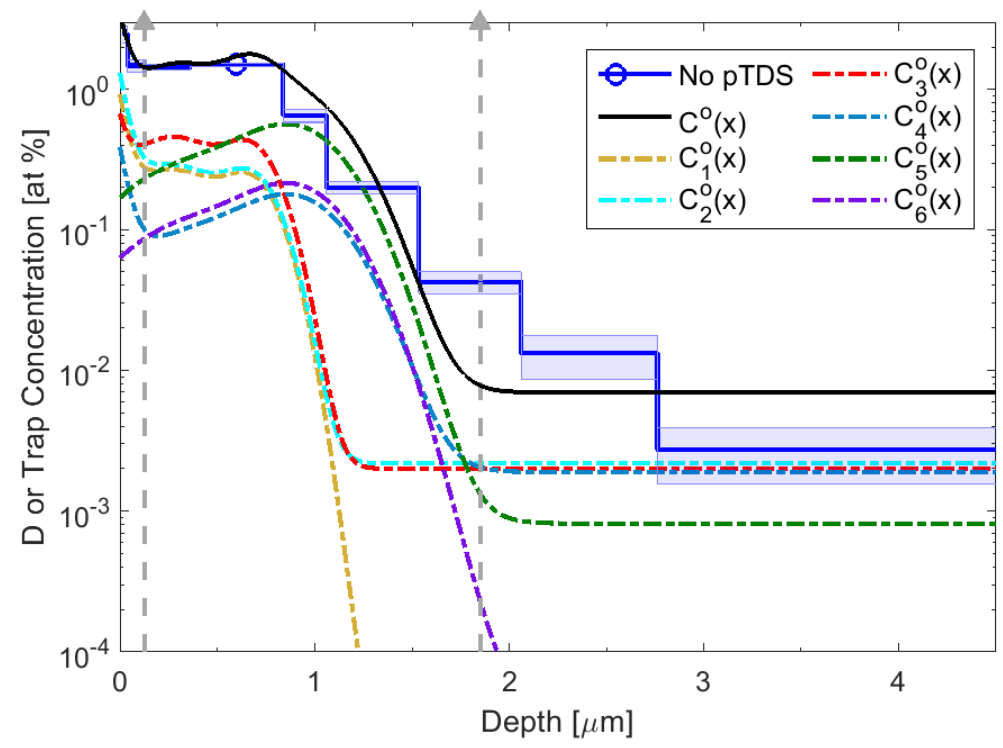

Figure 7: The total trap concentration (black) compared to the "No pTDS" NRA data (blue). The individual trap concentrations for $k=1-6$ are shown as dot-dashed lines.

instance, what appears as a single peak in Fig. 9 can be made of several nearby detrapping energies. If three detrapping energies made the peak near $620 \mathrm{~K}$, the lowest energy may be completely released, the middle partially, and the highest remained filled for a pTDS at $467 \mathrm{~K}$ (gold). As pointed out by Efimov et al. [13], several more intermediate pTDS peak-and-hold temperatures would be needed to discern if this was indeed the case.

\section{Discussion}

The detrapping energies found here are similar to values observed in previous studies as described in the Introduction. Some defects associated with the two lowest energies near 1.0 and $1.2 \mathrm{eV}$ are likely grain boundaries and dislocations as seen in the control sample without heavy ion damage. In ad- 


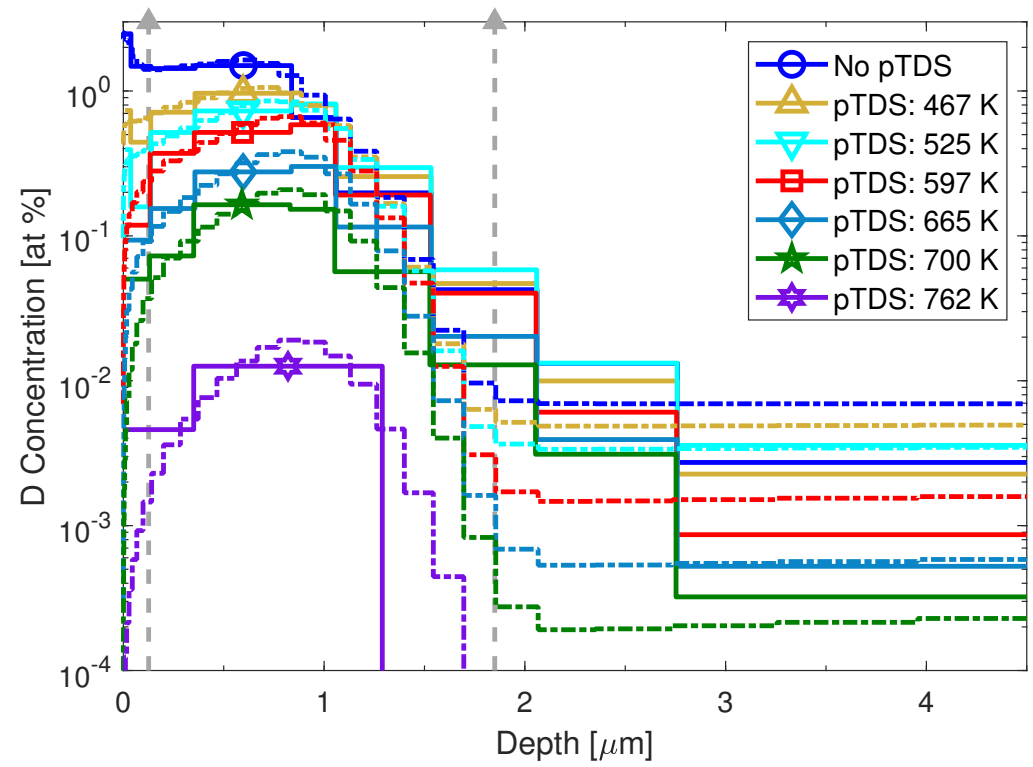

Figure 8: The comparison of the NRA data (solid) and the simulation result (dashed) using the optimized fit parameters, summarized in Table 2. For clarity, only 4 samples are shown.

dition, the spatial segregation of defects observed for the lowest three detrap energies of $1.0,1.2$, and $1.4 \mathrm{eV}$ are consistent with the multiple fill levels for mono-vacancies. The highest three detrap energies of 1.6, 1.8, and $1.9 \mathrm{eV}$ are likely related to vacancy clusters. The various overlapping values do not contradict, but demonstrates that nearby detrapping energies are not fully resolved.

Recent work done with x-ray spectroscopy [40] has quantified the number of defects due to dislocation loops. For a single crystalline W sample damaged to 0.2 dpa with $\mathrm{Cu}$ ions at $5 \mathrm{MeV}$, the vacancy-type dislocation loop concentration was near 0.1 at.\%. The scattering experiment was not spatially resolved but averaged over the first few microns of the $\mathrm{W}$ sur- 


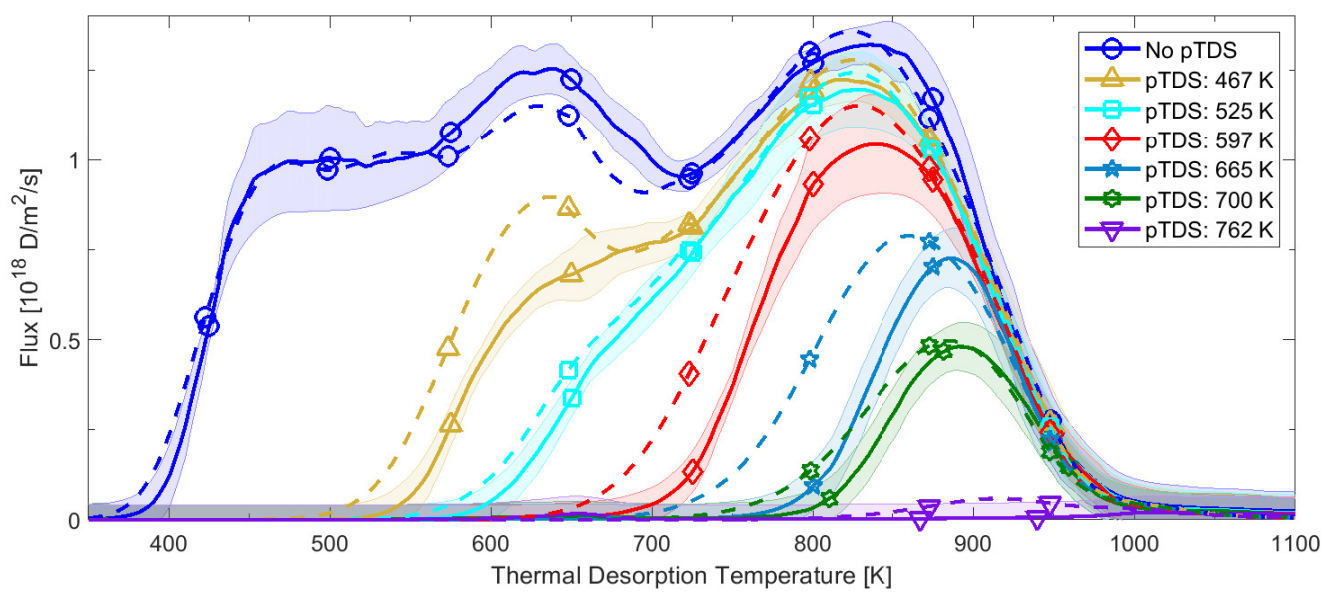

Figure 9: The comparison of the TDS data (solid) and the simulation result (dashed) using the optimized fit parameters, summarized in Table 2.

face, and could not resolve vacancy defects with radii below $5 \AA$. Thus that quoted vacancy concentration is for larger vacancy complexes averaged over the near-surface region and primarily includes large vacancy complexes. The $\mathrm{x}$-ray scattering results indicated that the average size of a vacancy complex was $(6.76 \pm 0.16) \AA$, which would correspond to a dislocation loop incorporating $\sim 30 \mathrm{~W}$ atoms. Traps 4-6 inferred in our work here have energies that are consistent with such larger vacancy complexes and have atomic concentrations of the same order of magnitude as these x-ray scattering results. Note that due to multiple occupancy, the concentration of D and traps are not likely one-to-one. The x-ray data also yields the size distribution, where the largest complexes are nearly two orders of magnitude lower in concentration than the smallest ( $5 \AA$ ) observable dislocation loop. Having the largest detrapping energy, the possible $7^{\text {th }}$ is likely due to a large vacancy complex and has a concentration nearly two order of magnitude lower than traps 4- 
6. This initial necessarily brief comparison between our inferred results and the x-ray scattering results thus shows a similar order-of-magnitude defect density and similar inferred defect size. Clearly additional work focused on direct measurement of defects, determination of the corresponding trapping energies from computational modeling and D atom capacity is needed in order to determine if the observed $\mathrm{D}$ retention and release is self-consistent with actual defect structures produced in the $\mathrm{W}$ material.

The spatial segregation of ion damage-induced defects shown in Fig. 5 suggest insights into how to compare D retention from ion beam damage with what might be expected to occur from neutron damage. While the neutron only interacts with the nuclei of lattice $W$, the electronic stopping loss of heavy ions produces a distinctly unique profile for various defects. The significantly higher Primary Knock-on Atom (PKA) energy for heavy ions will create larger and denser collision cascades as well as overlapping subcascades [41]. Molecular dynamic modeling by Sand et al. [42] predicts no subcascade break-up below $150 \mathrm{keV}$. A threshold energy for break-up near $150 \mathrm{keV}$ is predicted in [8] by comparing the similarity of $\mathrm{D}$ retention in neutron and heavy ion damaged $\mathrm{W}$. Though the maximum heavy ion PKA energy $(\sim \mathrm{MeV})$ significantly exceeds the neutron PKA energy $(\sim 300 \mathrm{keV})$, the threshold energy makes the sub-cascades comparable. Yet for heavy ions, the overlap of sub-cascades will increase and allow for more defect evolution (e.g. cluster formation) to occur as the cascades approach the Bragg peak. Our results show evidence for a significant deviation in the spatial location of high and low detrapping energies. The defects with higher detrapping energies (traps $k=4-6$ ) correlate to the SRIM predicted Bragg peak while 
defects with low detrapping energies (e.g. mono-vacancies and small vacancy clusters) are located between the surface and the Bragg peak location. Noting that the neutron damage profile in PFMs should yield similar mono-vacancy and small vacancy clusters, we are then led to speculate that retention and release from such neutron-induced damage might appear similar to what we observe in the intermediate spatial region of our experiments.

Finally we note that SRIM is a kinetic Monte-Carlo code that does not simulate the accumulation or agglomeration of defects [43]. Each simulated energetic ion interacts with a new, undisturbed amorphous target that does not retain the induced damage from previous ions and thus has no memory. The formation of defect structures such as loops or clusters is not accounted for as only displacements are counted when collisions impart enough energy to a lattice atom (i.e. above the displacement threshold). Qualitatively, SRIM predicts that the density of collision cascades increases as the heavy ion projectile loses energy to recoils and the highest density occurs at the peak dpa depth. Closer to the surface, the initially highly energetic heavy ions impart less energy to lattice atoms and are more likely to form smaller collision cascades nearer to the surface. The result may then be a segregation of defects, with more lower-order vacancies produced towards the surface and larger vacancy clusters closer to the peak dpa zone.

\section{Summary}

Trapped $\mathrm{D}$ in $\mathrm{Cu}$ ion damaged $\mathrm{W}$ was sequentially depopulated with increasing temperature to determine the spatial profile and detrapping energies. The total D retention measured through pTDS, NRA, and TDS are 
shown to be in excellent agreement as well as demonstrating the repeatability of sample preparation. Comparing the NRA data from the control sample, without pTDS, and the lowest pTDS temperatures demonstrates that all of the plasma induced defects in the near-surface were depopulated by holding the sample at $597 \mathrm{~K}$. In addition, the majority of retained D is depopulated by a pTDS temperature of $762 \mathrm{~K}$. For pTDS peak-and-hold temperatures between 525 and $762 \mathrm{~K}$, the D profiles measured via NRA are similar to the displacement damage profile predicted by SRIM. The full cycle of $\mathrm{D}_{2}$ plasma loading, to pTDS, and finally a final TDS cycle was modeled with TMAP7 utilizing a Pseudo Trap and Temperature Partition (PTTP) scheme. Detrapping energies near 1.0,1.2,1.4, 1.6, 1.8, and $1.9 \mathrm{eV}$ were found to fit the experimental data.

\section{Acknowledgments}

This work was supported by U.S. Department of Energy under DE-FG0207ER54912 and DE-SC0001999 as well as the University of California Office of Presidential Research Fund under 12-LR-237801. Part of this work has been carried out within the framework of the EUROfusion Consortium within the work project PFC and has received funding from the Euratom research and training programme 2014-2018 under grant agreement No 633053. The views and opinions expressed herein do not necessarily reflect those of the European Commission. 
579 9. Data Availability

${ }_{580}$ The raw/processed data required to reproduce these findings cannot be 581 shared at this time as the data also forms part of an ongoing study. Data 582 requests and inquiries may be sent to the primary author. 


\section{References}

[1] V. Philipps, Tungsten as material for plasma-facing components in fusion devices, Journal of Nuclear Materials 415 (2011) S2 - S9. Proceedings of the 19th International Conference on Plasma-Surface Interactions in Controlled Fusion.

[2] A. E521, Standard Practice for Neutron Radiation Damage Simulation by Charged-Particle Irradiation (1996). Reapproved 2009.

[3] O. V. Ogorodnikova, Fundamental aspects of deuterium retention in tungsten at high flux plasma exposure, Journal of Applied Physics 118 (2015) 074902.

[4] E. A. Hodille, A. Zalonik, S. Markelj, T. Schwarz-Selinger, C. S. Becquart, R. Bisson, C. Grisolia, Simulations of atomic deuterium exposure in self-damaged tungsten, Nuclear Fusion 57 (2017) 056002.

[5] W. Xiao, W. Geng, Role of grain boundary and dislocation loop in $\mathrm{h}$ blistering in $\mathrm{w}$ : A density functional theory assessment, Journal of Nuclear Materials 430 (2012) 132 - 136.

[6] K. Heinola, T. Ahlgren, K. Nordlund, J. Keinonen, Hydrogen interaction with point defects in tungsten, Phys. Rev. B 82 (2010) 094102.

[7] N. Fernandez, Y. Ferro, D. Kato, Hydrogen diffusion and vacancies formation in tungsten: Density functional theory calculations and statistical models, Acta Materialia 94 (2015) 307 - 318. 
[8] O. V. Ogorodnikova, V. Gann, Simulation of neutron-induced damage in tungsten by irradiation with energetic self-ions, Journal of Nuclear Materials 460 (2015) $60-71$.

[9] V. K. Alimov, B. Tyburska-Pschel, Y. Hatano, J. Roth, K. Isobe, M. Matsuyama, T. Yamanishi, The effect of displacement damage on deuterium retention in iter-grade tungsten exposed to low-energy, highflux pure and helium-seeded deuterium plasmas, Journal of Nuclear Materials 420 (2012) 370 - 373.

[10] A. Debelle, M. F. Barthe, T. Sauvage, First temperature stage evolution of irradiation-induced defects in tungsten studied by positron annihilation spectroscopy, Journal of Nuclear Materials 376 (2008) 216 - 221.

[11] S. Ryabtsev, Y. Gasparyan, M. Zibrov, A. Shubina, A. Pisarev, Deuterium thermal desorption from vacancy clusters in tungsten, Nuclear Instruments and Methods in Physics Research Section B: Beam Interactions with Materials and Atoms 382 (2016) 101 - 104. The 21st International workshop on Inelastic Ion Surface Collisions (IISC-21).

[12] M. H. J. 't Hoen, M. Mayer, A. W. Kleyn, H. Schut, P. A. Z. van Emmichoven, Reduced deuterium retention in self-damaged tungsten exposed to high-flux plasmas at high surface temperatures, Nuclear Fusion 53 (2013) 043003.

[13] V. S. Efimov, Y. M. Gasparyan, A. A. Pisarev, Investigation of a fine structure of deuterium thermal desorption spectra from tungsten, Jour- 
nal of Surface Investigation. X-ray, Synchrotron and Neutron Techniques 7 (2013) 472-478.

[14] F. Ferroni, X. Yi, K. Arakawa, S. P. Fitzgerald, P. D. Edmondson, S. G. Roberts, High temperature annealing of ion irradiated tungsten, Acta Materialia 90 (2015) $380-393$.

[15] M. Zhao, Q. Zhou, M. Nakata, A. Togari, F. Sun, Y. Hatano, N. Yoshida, Y. Oya, Deuterium removal efficiency in tungsten as a function of hydrogen ion beam fluence and temperature, in: G. Laukaitis (Ed.), Recent Advances in Technology Research and Education, Springer International Publishing, Cham, 2019, pp. 20-27.

[16] A. Zalonik, S. Markelj, T. Schwarz-Selinger, K. Schmid, Deuterium atom loading of self-damaged tungsten at different sample temperatures, Journal of Nuclear Materials 496 (2017) 1 - 8.

[17] M. J. Simmonds, Y. Q. Wang, J. L. Barton, M. J. Baldwin, J. H. Yu, R. P. Doerner, G. R. Tynan, Reduced deuterium retention in simultaneously damaged and annealed tungsten, Journal of Nuclear Materials 494 (2017) $67-71$.

[18] T. Schwarz-Selinger, Deuterium retention in mev self-implanted tungsten: Influence of damaging dose rate, Nuclear Materials and Energy 12 (2017) 683 - 688. Proceedings of the 22nd International Conference on Plasma Surface Interactions 2016, 22nd PSI.

[19] J. L. Barton, Y. Q. Wang, T. Dittmar, R. P. Doerner, G. R. Tynan, Deuterium retention in tungsten after heavy ion damage and hydro- 
gen isotope exchange in \{PISCES\}, Nuclear Instruments and Methods in Physics Research Section B: Beam Interactions with Materials and Atoms 332 (2014) $275-279$.

[20] R. E. Stoller, M. B. Toloczko, G. S. Was, A. G. Certain, S. Dwaraknath, F. A. Garner, On the use of $\{$ SRIM $\}$ for computing radiation damage exposure, Nuclear Instruments and Methods in Physics Research Section B: Beam Interactions with Materials and Atoms 310 (2013) 75 - 80.

[21] G. R. Tynan, A. D. B. III, G. A. Campbell, R. Charatan, A. de Chambrier, G. Gibson, D. J. Hemker, K. Jones, A. Kuthi, C. Lee, T. Shoji, M. Wilcoxson, Characterization of an azimuthally symmetric helicon wave high density plasma source, Journal of Vacuum Science \& Technology A 15 (1997) 2885-2892.

[22] K. J. Taylor, S. Yun, G. R. Tynan, Control of plasma parameters by using noble gas admixtures, Journal of Vacuum Science \& Technology A: Vacuum, Surfaces, and Films 22 (2004) 2131-2138.

[23] M. Mayer, E. Gauthier, K. Sugiyama, U. von Toussaint, Quantitative depth profiling of deuterium up to very large depths, Nuclear Instruments and Methods in Physics Research Section B: Beam Interactions with Materials and Atoms 267 (2009) 506 - 512.

[24] K. Schmid, U. von Toussaint, Statistically sound evaluation of trace element depth profiles by ion beam analysis, Nuclear Instruments and Methods in Physics Research Section B: Beam Interactions with Materials and Atoms 281 (2012) $64-71$. 
[25] J. H. Yu, M. J. Simmonds, M. J. Baldwin, R. P. Doerner, Deuterium desorption from tungsten using laser heating, Nuclear Materials and Energy (In Press) (2016).

[26] O. V. Ogorodnikova, J. Roth, M. Mayer, Deuterium retention in tungsten in dependence of the surface conditions, Journal of Nuclear Materials 313-316 (2003) 469 - 477. Plasma-Surface Interactions in Controlled Fusion Devices 15.

[27] R. Bisson, S. Markelj, O. Mourey, F. Ghiorghiu, K. Achkasov, J.-M. Layet, P. Roubin, G. Cartry, C. Grisolia, T. Angot, Dynamic fuel retention in tokamak wall materials: An in situ laboratory study of deuterium release from polycrystalline tungsten at room temperature, Journal of Nuclear Materials 467, Part 1 (2015) 432 - 438.

[28] M. J. Simmonds, J. H. Yu, Y. Q. Wang, M. J. Baldwin, R. P. Doerner, G. R. Tynan, Expanding the capability of reaction-diffusion codes using pseudo traps and temperature partitioning: Applied to hydrogen uptake and release from tungsten, Journal of Nuclear Materials 508 (2018) 472 $-480$.

[29] O. V. Ogorodnikova, J. Roth, M. Mayer, Ion-driven deuterium retention in tungsten, Journal of Applied Physics 103 (2008) 034902.

[30] M. Poon, A. A. Haasz, J. W. Davis, Modeling deuterium release during thermal desorption of $\mathrm{d}+$-irradiated tungsten, Journal of Nuclear Materials 374 (2008) $390-402$. 
[31] A. Rusinov, Y. Gasparyan, N. Trifonov, A. Pisarev, S. Lindig, M. Sakamoto, Investigation of hydrogen-defect interaction in tungsten by the probe fluence method, Journal of Nuclear Materials 415 (2011) S645 - S648. Proceedings of the 19th International Conference on Plasma-Surface Interactions in Controlled Fusion.

[32] B. Merrill, J. Jones, D. Holland, Tmap/mod 1: Tritium migration analysis program code description and user's manual, Idaho National Engineering Laboratory EGG-EP-7407, EG and G (1986).

[33] G. Longhurst, Tmap7 user manual, Idaho National Engineering and Environment Laboratory INEEL/EXT-04-02352(Rev. 2) (2008).

[34] J. Ambrosek, G. R. Longhurst, Verification and validation of tmap7, Idaho National Engineering and Environment Laboratory INEEL/EXT04-01657 (Rev. 2) (2008).

[35] M. A. Pick, K. Sonnenberg, A model for atomic hydrogen-metal interactions application to recycling, recombination and permeation, Journal of Nuclear Materials 131 (1985) 208 - 220.

[36] W. Eckstein, Calculated sputtering, reflection and range values, IPPReport IPP 9/132 (2002).

[37] L. Gao, W. Jacob, U. von Toussaint, A. Manhard, M. Balden, K. Schmid, T. Schwarz-Selinger, Deuterium supersaturation in lowenergy plasma-loaded tungsten surfaces, Nuclear Fusion 57 (2017) 016026. 
[38] S. Sakurada, K. Yuyama, Y. Uemura, H. Fujita, C. Hu, T. Toyama, N. Yoshida, T. Hinoki, S. Kondo, M. Shimada, D. Buchenauer, T. Chikada, Y. Oya, Annealing effects on deuterium retention behavior in damaged tungsten, Nuclear Materials and Energy 9 (2016) 141 - 144.

[39] S. Kirkpatrick, C. D. Gelatt, M. P. Vecchi, Optimization by Simulated Annealing, Science 220 (1983) 671-680.

[40] P. Sun, Y. Wang, M. Frost, C. Schnwlder, A. L. Levitan, M. Mo, Z. Chen, J. B. Hastings, G. R. Tynan, S. H. Glenzer, P. Heimann, Characterization of defect clusters in ion-irradiated tungsten by x-ray diffuse scattering, Journal of Nuclear Materials (2018).

[41] P. Vladimirov, S. Bouffard, Displacement damage and transmutations in metals under neutron and proton irradiation, Comptes Rendus Physique 9 (2008) 303 - 322. Materials subjected to fast neutron irradiation.

[42] A. E. Sand, S. L. Dudarev, K. Nordlund, High-energy collision cascades in tungsten: Dislocation loops structure and clustering scaling laws, EPL (Europhysics Letters) 103 (2013) 46003.

[43] J. Ziegler, J. Biersack, U. Littmark, The stopping and range of ions in matter, Pergamon Press (1985). 